\title{
Massachusetts health reform and disparities in joint replacement use: difference in differences study
}

\author{
(c) (1) (8) OPEN ACCESS
}

\author{
Amresh D Hanchate assistant professor ${ }^{1}$, Alok Kapoor assistant professor ${ }^{2}$, Jeffrey N Katz professor ${ }^{3}$, \\ Danny McCormick associate professor ${ }^{4}$, Karen E Lasser associate professor ${ }^{5}$, Chen Feng research \\ analyst ${ }^{6}$, Meredith G Manze visiting assistant professor ${ }^{7}$, Nancy R Kressin professor ${ }^{1}$
}

\begin{abstract}
${ }^{1}$ VA Boston Healthcare System and Boston University School of Medicine, Boston, MA, USA; ${ }^{2}$ University of Massachusetts Medical School, Worcester, MA, USA; ${ }^{3 B}$ Brigham and Women's Hospital and Harvard Medical School, Boston, MA USA; ${ }^{4}$ Cambridge Health Alliance and Harvard Medical School, Cambridge, MA, USA; ${ }^{5}$ Boston Medical Center and Boston University School of Medicine, Boston, MA, USA; ${ }^{6}$ Boston Medical Center, Boston, MA, USA ${ }^{7}$ City University of New York, School of Public Health, New York, NY, USA
\end{abstract}

\begin{abstract}
Objective To estimate the impact of the insurance expansion in 2006 on use of knee and hip replacement procedures by race/ethnicity, area income, and the use of hospitals that predominantly serve poor people ("safety net hospitals").
\end{abstract}

Design Quasi-experimental difference in differences study examining change after reform in the share of procedures performed in safety net hospitals by race/ethnicity and area income, with adjustment for patients' residence, demographics, and comorbidity.

Setting State of Massachusetts, United States.

Participants Massachusetts residents aged 40-64 as the target beneficiaries of reform and similarly aged residents of New Jersey, New York, and Pennsylvania as the comparison (control) population.

Main outcomes measures Number of knee and hip replacement procedures per 10000 population and use of safety net hospitals. Procedure counts from state discharge data for 2.5 years before and after reform, and multivariate difference in differences. Poisson regression was used to adjust for demographics, economic conditions, secular time, and geographic factors to estimate the change in procedure rate associated with health reform by race/ethnicity and area income.

Results Before reform, the number of procedures (/10 000) in Massachusetts was lower among Hispanic people $(12.9, \mathrm{P}<0.001)$ than black people (28.1) and white people (30.1). Overall, procedure use increased $22.4 \%$ during the 2.5 years after insurance expansion; reform in Massachusetts was associated with a $4.7 \%$ increase. The increase associated with reform was significantly higher among Hispanic people (37.9\%, $\mathrm{P}<0.001)$ and black people $(11.4 \%, \mathrm{P}<0.05)$ than among white people (2.8\%). Lower income was not associated with larger increases in procedure use. The share of knee and hip replacement procedures performed in safety net hospitals in Massachusetts decreased by $1.0 \%$ from a level of $12.7 \%$ before reform. The reduction was larger among Hispanic people $(-6.4 \%, \mathrm{P}<0.001)$ than white people $(-1.0 \%)$, and among low income residents $(-3.9 \%, p<0.001)$ than high income residents $(0 \%)$

Conclusions Insurance expansion can help reduce disparities by race/ethnicity but not by income in access to elective surgical care and could shift some elective surgical care away from safety net hospitals.

\section{Introduction}

Even in high income nations, out-of-pocket costs are a barrier to receipt of recommended medical care, particularly among low income and racial/ethnic minority populations. ${ }^{1-3}$ A survey of 11 countries in western Europe and North America found that the proportion of the population that did not get recommended medical care because of cost was highest in the United States (37\%) and the Netherlands (22\%) and lowest in the United Kingdom (4\%) and in Sweden (6\%). ${ }^{4}$ The historic Patient Protection and Affordable Care Act ("Obamacare") is a major policy intervention in the US aimed at reducing this barrier by extending health insurance coverage to over 30 million of the 51 million uninsured people. ${ }^{5}$ The target population is those aged 18-64, as the other age groups are nearly universally covered by public programs such as Medicare for adults aged 65 and older. In 2013, the national rate of uninsured people among those aged 18-64 was $18 \%$ but was considerably higher among Hispanic people (31\%), black people (21\%), and poor people $(31 \%){ }^{6}$ The extent to which the national insurance expansion will reduce disparities in access to healthcare is unclear. Lack of health insurance is associated 
with a wide range of disparities in utilization of healthcare, including the use of elective inpatient surgical procedures..$^{7-10}$ There is widespread evidence that use of elective procedures for various health problems, including cardiovascular, cancer, musculoskeletal, and digestive disorders, is significantly lower among minority and low income populations. ${ }^{1-13}$ The extent to which lack of insurance is causally associated with disparities in use of elective procedures is unclear ${ }^{7}$; lack of insurance is confounded with a range of other barriers to care, including low health literacy and difficulty in finding primary care physicians.

We examined this causal association by using data from a similar reform in the state of Massachusetts. Implemented in 2006-07, the Massachusetts health reform served as a template for the national law, sharing the three major elements of insurance expansion. ${ }^{14}$ First, it expanded all benefits of the public insurance coverage program, Medicaid, for adults with incomes below $133 \%$ of the federal poverty level (FPL). Second, it created a subsidized health insurance program, Commonwealth Care, for those with incomes between $133 \%$ and $300 \%$ federal poverty level. Finally, it introduced a penalty enforced mandate requiring virtually all state residents to have health insurance. One of the stated goals of the Massachusetts reform law was to reduce race based disparities in healthcare. ${ }^{15}$ The reform swiftly narrowed the race/ethnicity gap in coverage rates in Massachusetts. Whereas the overall rate of uninsurance fell from $8.4 \%$ (2006) to $3.3 \%$ (2008) among adults aged under $65,{ }^{16}$ this reduction was larger among black people (15\% to $5 \%$ ), Hispanic people ( $20 \%$ to $13 \%$ ), and those with lower incomes (18\% to $9 \%) .{ }^{17}$ In contrast with the overall reduction in uninsurance, evidence on improvements in access for minorities and people with low income has been mixed. Some prior research with patient surveys showed a reduction in uninsurance and increase in access to outpatient care overall but did not examine reform's effect on disparities in access to care or control for contemporaneous changes in utilization unrelated to health reform (secular changes). ${ }^{18}$ Others found declines in access to care by race/ethnicity with adjustment for secular trends by using self reported data. ${ }^{19}$ Studies that used objective data on health services utilization, including inpatient admissions, emergency room visits, and dental care, suggest little or modest improvement in access in Massachusetts but did not examine differential utilization by income or race/ethnicity. ${ }^{8-22}$

We previously examined post-reform access to care for adults aged under 65 using actual utilization records, measuring the differential change in utilization of a wide range of elective surgical procedures for disadvantaged populations. ${ }^{9}$ We found a significant increase in procedure use after reform among Hispanic people, white people, and populations from low and medium income areas, using Massachusetts patients aged 65 and older as controls. Use of a control population of a different age group (but the same state) from that of the cases, however, limited the ability to adjust for secular changes; also, our inclusion of a heterogeneous group of surgeries had uncertain implications for clinical value and cost effectiveness.

To deal with these limitations, we conducted new research focused on race/ethnicity and income disparities in utilization of inpatient surgeries for knee and hip replacement in Massachusetts patients aged under 65 compared with controls of the same age group in three sociodemographically similar states. ${ }^{23}$ Both are common and primarily elective procedures that have been used as markers of access to outpatient care. ${ }^{24}$ We also examined whether reform shifted use of these procedures away from hospitals that predominantly serve the uninsured and poor people ("safety net hospitals") in favor of non-safety net hospitals based on convenience to the patient, preference, and perceived quality. ${ }^{25}{ }^{26}$ Although these procedures are proved to be clinically and cost effective, ${ }^{27-29}$ evidence has documented large and persistent differences in joint replacement by race/ethnicity and income in the US and other countries, including Australia, Canada, and England. ${ }^{11-36}$ Use of both procedures has increased sharply among adults under 65 , aided by technological advances in the implants, which have broadened the indications for use in younger adults. ${ }^{36-38}$ In 2010 , patients under 65 received $45 \%$ of knee and $39 \%$ of hip replacements performed in the US. ${ }^{39} \mathrm{We}$ posited that insurance expansion would increase the overall use of joint replacement, especially among lower income patients and those of minority race/ethnicity.

\section{Methods}

\section{Data and study population}

We used comprehensive inpatient discharge data for Massachusetts and three comparison states (New Jersey, New York, and Pennsylvania) from 2004 to 2010 to identify all discharges for patients aged 40-64 with a primary total knee replacement or hip replacement using ICD-9 (international classification of diseases, ninth revision) codes 81.54 (total knee replacement) and 81.51 (total hip replacement). We excluded revision joint procedures because indications for such procedures can vary. ${ }^{31-40}$ To minimize bias associated with heterogeneity of surgical indication, we excluded patients aged under 40 and those with codes indicating infection of the knee or hip, metastatic or bone cancer, conversion of hemi-arthroplasty or other hip surgery to hip replacement, and fracture of the hip or femur. ${ }^{3140}$ We excluded people aged over 65 as inclusion would confound the impact of health insurance expansion with the impact of changes arising in Medicare insurance. We also excluded patients who were not residents of the state where the hospital was located because we wanted to calculate procedure rates for the resident state population. In identifying comparison states, we selected New Jersey, New York, and Pennsylvania for their geographic proximity and sizable minority populations. To estimate population rates we used census state level population data specific to sex, age, race/ethnicity, and year.

\section{Subpopulations: race/ethnicity and income}

Based on information in hospital discharge records, we grouped all patients into four race/ethnicity groups: Hispanic, non-Hispanic white, non-Hispanic black, and others. ${ }^{51}$ Data on race/ethnicity were missing for a small proportion of eligible patients $(1.1 \%$ in Massachusetts and $1.9 \%$ in the comparison states; see appendix tables A-C for additional details). We also grouped patients by income, based on the median income of patients' home zip code divided into three groups: low (lowest quarter of zip codes), medium (second lowest quarter) and high (top two quarters). ${ }^{52}$

\section{Safety net hospitals}

Following previous work, we defined safety net hospitals as the top quarter of hospitals in Massachusetts by the proportion of all inpatient admissions in the period before reform with the primary payer identified as self paying, Medicaid, or Free Care (a publicly funded program for the uninsured). ${ }^{43}$

\section{Periods before and after reform}

We treated the time period during which reform was introduced in Massachusetts (1 July 2006 to 31 December 2007) as the transition period; periods before and after reform were defined 
as the time before (1 January 2004 to 30 June 2006) and after (1 January 2008 to 30 June 2010) the transition period. ${ }^{44}$

\section{Outcomes}

Our primary outcome was the number of procedures per 10000 person years for adults aged 40-64 ("procedure rate"). The secondary outcome was the receipt of joint replacement procedures in safety net versus non-safety net hospitals for patients in Massachusetts.

\section{Analytic data}

To examine the primary outcome by race/ethnicity, we developed an analytic dataset based on aggregation of all patient observations by state of residence (four states), age (grouped in five categories), sex, time period (26 quarters), and race/ethnicity (four categories) into 4160 observations. For analysis by socioeconomic status, we used subgroups by income of zip code (three categories) in place of race/ethnicity, leading to 3120 observations. From the national population census, we obtained total population counts corresponding to each aggregated unit of observation.

\section{Analysis}

We estimated age and sex standardized procedure rates per 10 000 person years by race/ethnicity and area income for the periods before and after reform and longitudinally for each quarter from 2004 through $2010 .{ }^{45}$ Log linear interrupted time series models of quarterly procedure rates were estimated to obtain changes in procedure rate $(\%)$ by period and cohort. For the core difference in difference analysis, we estimated a multivariate Poisson regression model using the analytic dataset with race/ethnicity cohorts; the estimate of the change after reform in procedure rates in Massachusetts associated with health reform was obtained from the coefficient of the (interaction) indicator for Massachusetts residents in the period after reform..$^{21-46}$ This is interpreted as the extent to which change in Massachusetts exceeds that in the control states ("net change"). The regression model also adjusts for transition period changes. Net change for subpopulations by race/ethnicity and area income was based on extension of the Poisson models to include interaction terms with each race/ethnicity or area income subgroup (that is, estimation of difference in difference in differences). ${ }^{46}$ The estimates were adjusted for changes in the demographic composition (age and sex) in the census population. We also adjusted for persistent (time invariant) differences by geography with state level fixed effects and for secular temporal fluctuations with time fixed effects. ${ }^{21}{ }^{47} \mathrm{We}$ obtained robust standard error estimates adjusted for clustering of cohorts in each state. ${ }^{48}$ Significance was assessed at $\mathrm{P}<0.05$. All estimation was performed with Stata version 12.1. ${ }^{49}$

We calculated adjusted estimates of the net change in Massachusetts associated with reform for knee and hip replacements together and discretely and for subpopulations by race/ethnicity and area income.

We performed a secondary analysis to estimate the change in the proportion of procedures obtained at safety net hospitals in Massachusetts using individual discharge as the unit of analysis. For comparability of patterns before and after reform, and to account for differences in the demographics and location of patients, we constructed logistic regression models, separately by race/ethnicity and income cohorts, of the dichotomous indicator of receipt of a knee or hip replacement procedure at a safety net hospital, based on records before reform, and using as predictors, patient age, sex, comorbidity (Charlson score), and the difference in distance between home zip code to the nearest safety net hospital and to the nearest non-safety net hospital. ${ }^{26}$ For each cohort, we applied the model estimates to discharge records after reform and estimated the expected proportion of procedures to be performed in safety net hospitals and compared it with the observed proportion.

\section{Sensitivity analysis}

We examined sensitivity of the main findings to expanding the study population to those aged 18-64 and adding state level unemployment (quarterly) to adjust for differences in the economic downturn during the study period. To examine for a linear trend in the association between income and reform effect, we estimated the main model treating income category as a continuous measure.

\section{Results}

Between the periods before and after reform, the volume of knee and hip replacement procedures increased by $37 \%$ in Massachusetts and $29 \%$ in comparison states (table $1 \Downarrow$ ). Across all states, knee replacements accounted for $64 \%$ and $65 \%$ of the combined total of procedures in the periods before and after reform, respectively (see appendix table D).

The proportion of the overall volume of knee and hip replacement procedures received by black and Hispanic people (combined) increased from $6.4 \%$ (before reform) to $8.0 \%$ (after reform) in Massachusetts and from $13.1 \%$ to $13.3 \%$ in the comparison states. Similarly, the proportion of procedures received by residents of low income areas increased from 19.9\% to $21.0 \%$ in Massachusetts and from $20.0 \%$ to $20.4 \%$ in the comparison states. Estimates of time series quarterly changes in rates of procedures (per 10000 person years), adjusted for age and sex, indicated that minority and low income area residents in Massachusetts experienced larger increases after the reform than their counterparts in the comparison states (figure $\downarrow$ ); in addition, the extent to which rates in Massachusetts exceeded those in the comparison states in the period after reform was larger for racial/ethnic minorities (14\%) than for white people $(7 \%, \mathrm{P}<0.001)$ and low income area residents (16\%) compared with high income area residents $(4 \%, \mathrm{P}<0.01)$.

\section{Baseline differences in procedure rates by race/ethnicity and area income}

In Massachusetts, the rate of knee and hip replacement procedures for Hispanic people (12.9 procedures) before reform was $57 \%$ lower than the rate for white people (30.1 procedures; $\mathrm{P}<0.001$ ) (table $2 \Downarrow$ ). Similarly, the rate for residents of low income areas (25.5 procedures) was $16 \%$ lower than the rate for residents of high income areas $(30.4$ procedures, $\mathrm{P}<0.001)$. This pattern was also found for knee and hip replacement procedures; further, rates of hip replacement were significantly (21\%) lower for black people than for white people.

\section{Changes after reform in procedure rates by race/ethnicity and area income}

Across Massachusetts, use of knee and hip replacements increased between the periods before and after reform by $22.4 \%$ (table $2 \Downarrow$ ). After adjustment for secular changes (such as those observed in comparison states), the estimated net increase associated with health reform was $4.7 \%$ (95\% confidence interval $2.3 \%$ to $7.1 \%$ ). The net increase was significantly higher among Hispanic $(37.9 \%, \mathrm{P}<0.001)$ and black people $(11.4 \%$, $\mathrm{P}=0.03)$ than among white people $(2.8 \%)$. This pattern was also 
found for knee and hip replacement procedures individually. Whereas residents of low income areas experienced higher net increases for both procedures compared with their high income area counterparts, the differences were not significant. These findings remained robust in the sensitivity analysis (see appendix table E); we found no significant linear trend in the association between income and net change in procedure use.

\section{Changes after reform in use of safety net hospitals}

Safety net hospitals accounted for $12.7 \%$ of knee and hip replacement procedures in Massachusetts (table $3 \Downarrow$ ); the number of safety net hospitals remained unchanged before and after reform (see appendix table F). Use of safety net hospitals for knee and hip replacement procedures varied across subgroups. Before reform, the proportion of the procedures obtained in safety net hospitals was significantly higher among Hispanic (30.3\%) and black people (30.4\%) than white people (11.5\%). The proportion was higher for low income area residents $(25.3 \%)$ than for high income area residents $(8.0 \%)$. Many groups shifted care to non-safety net hospitals during the period after reform. Overall, the proportion of procedures carried out in safety net hospitals decreased from $12.7 \%$ (before reform) to $12.1 \%$ (after reform); after adjustment for patient demographics and residential location, the proportion of procedures performed at safety net hospitals decreased by $1.0 \%$ $(\mathrm{P}<0.001)$. The largest decrease was among Hispanic people $(-6.4 \%)$ and residents of low income areas $(-3.9 \%)$. Although the proportion of procedures at safety net hospitals decreased for black people ( $30.4 \%$ to $28.3 \%$ ), the change, after adjustment, was not significant.

\section{Discussion}

\section{Principal findings}

The 2006 Massachusetts insurance expansion was associated with a significant increase in utilization of elective knee and hip replacement procedures after reform across several subpopulations. Hispanic and black people, groups with relatively larger gains in insurance after the expansion, experienced significantly higher rates of increase than white people. There was a modest shift away from safety net hospitals as the provider of these procedures, with a larger shift among Hispanic people and residents of low area incomes. We found no significant changes by area income.

\section{Comparison with other studies}

Our findings of increased utilization are consistent with previous reports of generally increased access, particularly for Hispanic people. ${ }^{19-51}$ Using self reported survey data, one study estimated that, among Hispanic people, the adjusted rate of having a usual source of healthcare increased from $77 \%$ (2005) to $86 \%$ (2009) and the rate of forgoing care because of cost decreased from $10.4 \%$ (2005) to $9.3 \%$ (2009). ${ }^{52}$ The same study reported that gains in insurance among Hispanic people were more than double those for non-Hispanic people and credited special programs aimed at monitoring and enrolling uninsured vulnerable populations. ${ }^{52}$ In our current analysis, reform accounted for $12 \%$ of the overall increase in procedure rates among white people and $98 \%$ of the increase among Hispanic people.

Although these findings echo those from our previous work on a heterogeneous group of 17 elective surgical procedures, ${ }^{9}$ there are notable differences. Specifically, in our earlier study we found no significant differences by race or ethnicity, but we found larger increases among Hispanic and black people than white people among this more homogenous set of joint replacement surgeries. In contrast with the present study, the previous study used Massachusetts residents aged $\geq 65$ as the comparison cohort; changes in practice patterns or Medicare payments affecting only the older group could have distorted the estimate of reform effect.

Our findings indicate that lack of health insurance is an additional factor to explain disparities in utilization of some elective surgical procedures. Previous studies among insured people found that lower rates of procedure use among minorities are associated with higher rates of fear of complications and lack of trust in effectiveness of treatment. ${ }^{30-54}$ Future studies should examine the relative contributions of insurance compared with other factors.

An estimated 411000 Massachusetts residents gained health insurance after reform, through Medicaid expansion, extended employer based private plans, or the newly established state subsidized plans available through the state insurance exchange.$^{55}$ Unlike in the period before reform, when safety net providers supplied inpatient care for the uninsured (with funding from the uncompensated care pool program), in the period after reform newly insured people gained freedom in their choice of providers. This could underlie our finding of a small but significant shift away from safety net hospitals, with the largest shift - a reduction in share of safety net hospitals from $53 \%$ to $48 \%$ - occurring among residents of low income areas. Previous work has indicated a substantial concentration of minority and poor patients in a small proportion of US hospitals, often bypassing more proximate and higher volume hospitals. ${ }^{26} \mathrm{~A}$ shift away from safety net hospitals after health reform indicates greater choice in hospital selection based on patients' convenience and preference; alternately, a shift to non-safety net hospitals could also arise from spillover of increased demand unmet at safety net hospitals. An exception, however, was seen among black people, for whom there was no shift in use of safety net hospitals; this pattern is consistent with previous findings of clustering of inpatient care for black people covered by Medicare to a limited range of hospitals. ${ }^{25}{ }^{26}$ Note that procedure volumes increased in both safety net hospitals and non-safety net hospitals, but the increase was larger in the latter group.

Our findings also indicate a sizable secular increase in procedure use in all the states examined. This reflects an increasing trend, particularly among younger adults, nationwide as well as in other countries, including Canada, the UK, Germany, and Switzerland. ${ }^{38-58}$ While there is no definitive explanation for this trend, conjectures include expansion of indications for these procedures and advances in implant technology. ${ }^{37-56}$

\section{Implications}

Our findings indicate that expanded insurance coverage has considerable potential for amelioration of disparities related to race and income in use of elective surgery at the national level in the US. The insurance expansion directed by the Patient Protection and Affordable Care Act is currently underway with expansion of Medicaid, subsidized insurance through exchanges, and an individual mandate to have insurance. Effects will likely vary across states because of wide differences in baseline uninsurance rates, share of racial/ethnic minorities, provider availability, and uptake of Medicaid expansion, in addition to anticipated differences in implementation of expansion, compliance, and enforcement of mandates. ${ }^{59}$ Compared with the baseline rate of uninsurance among non-elderly adults in 
Massachusetts (8.4\% in 2006), the corresponding US rate is considerably higher (18\% in 2013), with a wide range across states (11\% to $31 \%$ in 2013 ); in some states, the proportion of minorities among uninsured people nears $60 \%$ for Hispanic people (in Texas and California) and exceeds $30 \%$ for black people (in Alabama, Georgia, and Louisiana). ${ }^{6}$ Moreover, the availability of physician providers (primary care and specialty) is considerably higher in Massachusetts than most other states (46v 27 per 10000 inhabitants with 2011 estimates). ${ }^{6}$

More generally, cost barriers are a source of disparities in access to medical care in many countries ${ }^{34}$; as provision of health insurance is a major step in reducing patient costs, particularly for expensive elective procedures, our study suggests a causal connection between insurance expansion and reduced barriers to care. We also found that some minority groups shifted care from hospitals that primarily served poor people (safety net) to other hospitals, suggesting greater choice in terms of convenience and preference.

\section{Limitations}

Our study has several limitations. First, similar to other studies on the impact of Massachusetts health reform that use a difference in differences design, our findings are susceptible to confounding from changes in use of procedures unique to Massachusetts over the study period but independent of health reform. ${ }^{9-61}$ For instance, Massachusetts hospitals might be early adopters of new indications for a surgical procedure; however, as such trends would likely be evident across all racial/ethnic groups and income subpopulations in Massachusetts, our finding of decreases in racial/ethnic disparities is robust to this potential confounding. Second, the administrative data we analyzed do not provide clinical details on patient preferences or symptoms, imaging results, or appropriateness of procedure use. Cohort level differences in procedure rates can arise from systematic differences in the underlying incidence of arthritis and associated pain and functional limitations; future studies should examine trends in appropriateness and impact on patient outcomes by using detailed information on clinical status and patient functional health. ${ }^{35}$ Current radiologic evidence for adults aged $\geq 60$ from the US National Health and Nutrition Examination Survey (NHANES III, 1991-94), however, indicates that the prevalence of symptomatic osteoarthritis among black people $(17.7 \%)$ is (marginally) higher and among Hispanic people $(14.8 \%)$ is not significantly lower than that among white people $(11.9 \%){ }^{62}$ In addition, there is substantial previous evidence of underutilization of joint procedures by minorities unrelated to clinical need. ${ }^{30-63}$ We limited our study population to adults aged under 65 as this was the target beneficiary age group of the reform. Finally, as zip code is the finest level of patient location available in the data source, we were unable to use more specific area level (such as census block) indicators; however, zip code is sensitive for state or national data and has been used in previous studies. ${ }^{1164}$

\section{Conclusions}

Massachusetts health reform was associated with a significant increase in utilization of knee and hip replacement and a reduction in disparities related to race and ethnicity, suggesting improved access for minorities. These results suggest that insurance reform can be an important lever for reducing disparities in use of such procedures, although greater levels of uninsurance and shortages of primary care providers in the rest of the country present formidable challenges to achieving these reductions more widely.
We thank Lisa Marks who assisted with preparation of the manuscript. Contributors: All authors contributed to the data analysis and interpretation of the results, and reviewed and approved the final manuscript. ADH had full access to all of the data in the study and takes responsibility for the integrity of the data and the accuracy of the data analysis. ADH is guarantor.

Funding: This research was funded by US National Institutes of Health grants (1R21NS062677, ADH, principal investigator, and 1U01HL105342-01, NRK, principal investigator) and a grant from the $\mathrm{Rx}$ foundation. NRK is supported in part by a senior research career scientist award from the Department of Veterans Affairs, Health Services Research and Development Service (RCS 02-066-1). The views expressed in this article are those of the authors and do not necessarily represent the views of the National Institutes of Health, the Rx foundation, Boston University, or the Department of Veterans Affairs. Competing interests: All authors have completed the ICMJE uniform disclosure at www.icmje.org/coi_disclosure.pdf (available on request from the corresponding author) and declare: ADH, NRK, KEL, DM, AK, MGM, and CF received grants from National Institutes of Health, $R x$ Foundation, during the conduct of the study; no financial relationships with any organizations that might have an interest in the submitted work in the previous three years; no other relationships or activities that could appear to have influenced the submitted work.

Ethical approval: This study was approved by the Boston University Medical Campus institutional review board.

Data sharing: Inpatient data files were restricted for this project and cannot be shared because of restrictions on use of data. Statistical code files are available from the corresponding author.

Transparency: The lead author, ADH, affirms that the manuscript is an honest, accurate, and transparent account of the study being reported; that no important aspects of the study have been omitted; and that any discrepancies from the study as planned (and, if relevant, registered) have been explained.

1 Van Doorslaer E, Masseria C, Koolman X, Group ftOHER. Inequalities in access to medical care by income in developed countries. CMAJ 2006;174:177-83.

2 Rodin D, Stirbu I, Ekholm O, Dzurova D, Costa G, Mackenbach JP, et al. Educational inequalities in blood pressure and cholesterol screening in nine European countries. $J$ Epidemiol Community Health 2012;66:1050-5.

3 Diderichsen F, Andersen I, Manuel C, Working Group of Danish Review on Social Determinants of Health, Andersen AM, Bach E, et al. Health inequality-determinants and policies. Scan J Public Health 2012;40(8 suppl):12-105.

4 Davis K, Stremikis K, Squires D, Schoen C. Mirror, mirror on the wall: how the performance of the US health care system compares internationally. The Commonwealth Fund, 2014. www.commonwealthfund.org/ /media/files/publications/fund-report/2014/jun/1755_davis_ mirror_mirror_2014.pdf.

5 AHRQ. National healthcare disparities report 2013. Agency for Healthcare Research and Quality, 2013.

6 Kaiser Family Foundation. State health facts. 2014. www.statehealthfacts.org.

7 Levy H, Meltzer D. The impact of health insurance on Health. Ann Rev Public Health 2008;29:399-409.

8 Miller S. The effect of the Massachusetts reform on health care utilization. Inquiry 2012;49:317-26.

9 Hanchate AD, Lasser KE, Kapoor A, Rosen J, McCormick D, D'Amore MM, et al. Massachusetts reform and disparities in inpatient care utilization. Medical Care 2012;50:569-77.

10 Lasser KE, Hanchate AD, McCormick D, Manze MG, Chu C, Kressin NR. The effect of Massachusetts health reform on 30 day hospital readmissions: retrospective analysis of hospital episode statistics. BMJ 2014;348:g2329.

11 Jha AK, Fisher ES, Li Z, Orav EJ, Epstein AM. Racial trends in the use of major procedures among the elderly. N Engl J Med 2005;353:683-91.

12 Mensah GA, Mokdad AH, Ford ES, Greenlund KJ, Croft JB. State of disparities in cardiovascular health in the United States. Circulation 2005;111:1233-41.

13 Jemal A, Simard EP, Dorell C, et al. Annual report to the nation on the status of cancer, 1975-2009, featuring the burden and trends in human papillomavirus (HPV)-associated cancers and HPV vaccination coverage levels. J Natl Cancer Inst 2103;105:175-2012.

14 Levy H. Health reform: learning from Massachusetts. Inquiry 2012;49:300-2.

15 187th General Court of the Commonwealth of Massachusetts. Massachusetts General Laws, chapter 6A, section 160, Health Disparities Countil; duties; composition; meetings; annual reports. Boston, MA. www.malegislature.gov/Laws/GeneralLaws/Partl/Titlell/ Chapter6A/Section 160

16 Long SK, Phadera L. Estimates of health insurance coverage in Massachusetts from the 2009 Massachusetts health insurance survey. Urban Institute, 2009.

17 Massachusetts Department of Public Health. A profile of health among Massachusetts adults, 2006 \& 2009. Commonwealth of Massachusetts, 2010

18 Long SK, Stockley K, Dahlen H. Massachusetts health reforms: uninsurance remains low, self-reported health status improves as state prepares to tackle costs. Health Aff (Millwood) 2012;31:444-51. 


\section{What is already known on this topic}

Cost remains a barrier to obtaining needed medical care, even in high income countries, especially for major elective surgical procedures In the US, with $18 \%$ of the population aged 18-64 having no health insurance, large scale insurance coverage expansions have the potential to reduce these barriers, particularly among racial/ethnic minority and low income populations

\section{What this study adds}

Based on data from expansion of insurance coverage in Massachusetts' in 2006-07, there was a narrowing of racial/ethnic disparities in use of elective surgical care

There was a shift away from safety net hospitals, particularly among Hispanic and low income people, suggesting an increase in choice of providers

19 Pande AH, Ross-Degnan D, Zaslavsky AM, Salomon JA. Effects of healthcare reforms on coverage, access, and disparities: quasi-experimental analysis of evidence from Massachusetts. Am J Prev Med 2011;41:1-8.

20 Chen C, Scheffler G, Chandra A. Massachusetts' health care reform and emergency department utilization. N Engl J Med 2011;365:e25.

21 Kolstad JT, Kowalski AE. The impact of health care reform on hospital and preventive care: evidence from Massachusetts. J Public Econ 2012;96:909-29.

22 Nasseh K, Vujicic M. Health reform in Massachusetts increased adult dental care use particularly among the poor. Health Aff (Milwood) 2013;32:1639-45.

23 Cram P, Lu X, Kates SL, Singh JA, Li Y, Wolf BR. Total knee arthroplasty volume, utilization, and outcomes among medicare beneficiaries, 1991-2010. JAMA 2012;308:1227-36.

24 Billings J, Zeitel L, Lukomnik J, Carey TS, Blank AE, Newman L. Impact of socioeconomic status on hospital use in New York City. Health Aff (Millwood) 1993;12:162-73.

25 Jha AK, Orav EJ, Li Z, Epstein AM. Concentration and quality of hospitals that care for elderly black patients. Arch Intern Med 2007;167:1177-82.

26 Losina E, Wright EA, Kessler CL, Barrett JA, Fossel AH, Creel AH, et al. Neighborhoods matter: use of hospitals with worse outcomes following total knee replacement by patients from vulnerable populations. Arch Intern Med 2007;167:182-7.

27 Daigle ME, Weinstein AM, Katz JN, Losina E. The cost-effectiveness of total joint arthroplasty: a systematic review of published literature. Best Pract Res Clin Rheumatol 2012;26:649-58.

28 Dakin H, Gray A, Fitzpatrick R, MacLennan G, Murray D, Group TKT. Rationing of total knee replacement: a cost-effectiveness analysis on a large trial data set. BMJ Open 2012;2:e000332.

29 Losina E, Walensky RP, Kessler CL, et al. Cost-effectiveness of total knee arthroplasty in the United States: patient risk and hospital volume. Arch Intern Med 2009;169:1113-21.

30 Kane RL, Wilt T, Suarez-Almazor ME, Fu SS. Disparities in total knee replacements: a review. Arthritis Rheum 2007:57:562-7.

31 Mahomed NN, Barrett J, Katz JN, Baron JA, Wright J, Losina E. Epidemiology of total knee replacement in the United States Medicare population. J Bone Joint Surg Am 2005;87:1222-28

32 Mahomed NN, Barrett JA, Katz JN, Phillips CB, Losinia E, Lew RA, et al. Rates and outcomes of primary and revision total hip replacement in the United States medicare population. J Bone Joint Surg Am Jan 2003;85-A:27-32

33 Skinner J, Weinstein JN, Sporer SM, Wennberg JE. Racial, ethnic, and geographic disparities in rates of knee arthroplasty among Medicare patients. N Engl J Med 2003;349:1350-9.

34 Dixon T, Urquhart DM, Berry P, Bhatia K, Wang $\mathrm{Y}$, Graves $\mathrm{S}$, et al. Variation in rates of hip and knee joint replacement in Australia based on socio-economic status, geographical locality, birthplace and indigenous status. ANZ J Surg 2011;81:26-31.

35 Hawker GA, Wright JG, Coyte PC, Williams JI, Harvey B, Glazier R, et al. Determining the need for hip and knee arthroplasty: the role of clinical severity and patients' preferences. Med Care 2001;39:206-16

36 Judge A, Welton NJ, Sandhu J, Ben-Shlomo Y. Equity in access to total joint replacemen of the hip and knee in England: cross sectional study. BMJ 2010;341:c4092.

37 Losina E, Katz JN. Total knee arthroplasty on the rise in younger patients: are we sure that past performance will guarantee future success? Arthritis Rheum 2012:64:339-41.

38 Ravi B, Croxford R, Reichmann WM, Losina E, Katz JN, Hawker GA. The changing demographics of total joint arthroplasty recipients in the United States and Ontario from 2001 to 2007. Best Pract Res Clin Rheumatol 2012;26:637-47.

39 Agency for Healthcare Research and Quality. HCUPNet: A tool for identifying, tracking, and analyzing national hospital statistics. 2014. http://hcupnet.ahrq.gov.

40 Katz JN, Losina E, Barrett J, Phillips CB, Mahomed NN, Lew RA, et al. Association between hospital and surgeon procedure volume and outcomes of total hip replacement in the United States medicare population. J Bone Joint Surg Am 2001;83-A:1622-9.

41 Schoenman JA, Sutton JP, Kintala S, Love D, Maw R. The value of hospital discharge databases. NORC at the University of Chicago, 2005.

42 Methodology for the state and county resident population estimates by age, sex, race and Hispanic origin (Vintage 2008): April 1, 2000 to July 1, 2008. US Census Bureau, 2010

43 Werner RM, Goldman LE, Dudley RA. Comparison of change in quality of care between safety-net and non-safety-net hospitals. JAMA 2008;299:2180-7.
44 McDonough JE, Rosman B, Butt M, Tucker L, Howe LK. Massachusetts health reform implementation: major progress and future challenges. Health Aff (Millwood) 2008;27:w285-97.

45 Woodward M. Epidemiology: study design and data analysis. 2nd ed. Chapman and Hall/CRC, 2005.

46 Wooldridge JM. Econometric analysis of cross-section and panel data. MIT Press, 2002.

47 Hanchate AD, Zhang Y, Felson DT, Ash AS. Exploring the determinants of racial and ethnic disparities in total knee arthroplasty: health insurance, income, and assets. Med Care 2008;46:481-8.

48 Cameron AC, Trivedi PK. Microeconometrics: methods and applications. Cambridge University Press, 2005.

49 StataCorp. Stata statistical software: release 12. StataCorp LP, 2012.

50 Long SK, Stockley K, Nordahl KW. Coverage, access, and affordability under health reform: learning from the Massachusetts model. Inquiry 2012;49(4):303-316.

51 Zhu J, Brawarsky P, Lipsitz S, Huskamp H, Haas JS. Massachusetts health reform and disparities in coverage, access and health status. J Gen Intern Med 2010;25:1356-62.

52 Maxwell J, Cortés DE, Schneider KL, Graves A, Rosman B. Massachusetts' health care reform increased access to care for Hispanics, but disparities remain. Health Affairs 2011;30:1451-60.

53 Ibrahim SA, Stone RA, Han X, Cohen P, Fine MJ, Henderson WG, et al. Racial/ethnic differences in surgical outcomes in veterans following knee or hip arthroplasty. Arthritis Rheum 2005;52:3143-51.

54 Blum MA, Ibrahim SA. Race/ethnicity and use of elective joint replacement in the management of end-stage knee/hip osteoarthritis: a review of the literature. Clinic Geriatr Med 2012;28:521-32.

55 Massachusetts health reform: a five-year progress report. BCBSFo, 2011.

56 Losina E, Thornhill TS, Rome BN, Wright J, Katz JN. The dramatic increase in total knee replacement utilization rates in the United States cannot be fully explained by growth in population size and the obesity epidemic. J Bone Joint Surg Am 2012:94:201-7.

57 Culliford DJ, Maskell J, Beard DJ, Murray DW, Price AJ, Arden NK. Temporal trends in hip and knee replacement in the United Kingdom: 1991 to 2006. J Bone Joint Surg Br 2010;92:130-5.

58 Falbrede I, Widmer M, Kurtz S, Schneidmuller D, Dudda M, Roder C. [Utilization rates of lower extremity prostheses in Germany and Switzerland: a comparison of the years 2005-2008]. Orthopade 2011;40:793-801.

59 Sommers BD, Epstein AM. US Governors and the Medicaid expansion-no quick resolution in sight. N Engl J Med 2013;368:496-9.

60 Pear R. States' policies on health care exclude some of the poorest. New York Times 2103 May 24.

61 Sommers BD, Baicker K, Epstein AM. Mortality and access to care among adults afte state Medicaid expansions. N Engl J Med 2012;367:1025-34

62 Dillon CF, Rasch EK, Gu Q, Hirsch R. Prevalence of knee osteoarthritis in the United States: arthritis data from the Third National Health and Nutrition Examination Survey 1991-94. J Rheumatol 2006;33:2271-9.

63 Hawker GA, Wright JG, Coyte PC, Williams JI, Harvey B, Glazier R, et al. Determining the need for hip and knee arthroplasty: the role of clinical severity and patients' preferences. Med Care 2001:39:206-16.

64 Chernew M, Gibson TB, Yu-Isenberg K, Sokol MC, Rosen AB, Fendrick AM. Effects of increased patient cost sharing on socioeconomic disparities in health care. $J$ Gen Intern Med 2008;23:1131-6.

\section{Accepted: 19 December 2014}

\section{Cite this as: BMJ 2015;350:h440}

This is an Open Access article distributed in accordance with the Creative Commons Attribution Non Commercial (CC BY-NC 4.0) license, which permits others to distribute, remix, adapt, build upon this work non-commercially, and license their derivative works on different terms, provided the original work is properly cited and the use is non-commercial. See: http://creativecommons.org/licenses/by-nc/4.0/. 


\section{Tables}

Table 1| Population and procedure counts for joint replacement (knee and hip), 2004-10, in people aged 40-64 in Massachusetts and comparison states (New Jersey, New York, and Pennsylvania). Figures are numbers (percentage) unless stated otherwise

Massachusetts

Before reform, 2004-06* After reform, 2008-10†

Total No of procedures performed

\begin{tabular}{|c|c|c|c|c|}
\hline Women & $8278(55.1)$ & $11403(55.4)$ & $50411(59.4)$ & $63620(58.2)$ \\
\hline Men & $6741(44.9)$ & $9170(44.6)$ & 34439 (40.6) & $45785(41.8)$ \\
\hline \multicolumn{5}{|c|}{ Age (years): } \\
\hline $40-54$ & $5283(35.2)$ & $7290(35.4)$ & 30451 (35.9) & $37593(34.4)$ \\
\hline $55-64$ & $9746(64.9)$ & $13283(64.6)$ & $54399(64.1)$ & $71812(65.6)$ \\
\hline \multicolumn{5}{|c|}{ Race/ethnicity: } \\
\hline White & $13566(90.3)$ & $18451(89.7)$ & $69219(81.6)$ & $89822(82.1)$ \\
\hline Black & $680(4.5)$ & $1101(5.4)$ & $8111(9.6)$ & $10840(9.9)$ \\
\hline Hispanic & $279(1.9)$ & $541(2.6)$ & $2986(3.5)$ & $3773(3.4)$ \\
\hline Other & $257(1.7)$ & $328(1.6)$ & $2358(2.8)$ & $3463(3.2)$ \\
\hline Unknown & $237(1.6)$ & $152(0.7)$ & $2176(2.6)$ & $1,507(1.4)$ \\
\hline \multicolumn{5}{|c|}{ Zip code median incomeł: } \\
\hline Low & $2988(19.9)$ & $4315(21.0)$ & $16739(20.0)$ & 21919 (20.4) \\
\hline Medium & $3724(24.8)$ & $5044(24.6)$ & 22247 (26.5) & $28648(26.6)$ \\
\hline High & $8281(55.2)$ & $11178(54.4)$ & $44818(53.5)$ & $57121(53.0)$ \\
\hline
\end{tabular}

*From first quarter in 2004 to second quarter in 2006.

†From first quarter in 2008 to second quarter in 2010.

$\ddagger$ Area income could not be grouped in small proportion of cases $(0.2 \%$ in MA and $1.7 \%$ in comparisons states) because of missing or invalid zip code. 
Table 2| Rates of and changes in utilization of total knee and hip replacements after health reform in Massachusetts (MA) by race/ethnicity and socioeconomic status

\begin{tabular}{|c|c|c|c|c|c|}
\hline \multirow[b]{2}{*}{ Population } & \multicolumn{2}{|c|}{ Procedure rate $(/ 10000)(95 \% \mathrm{Cl})$} & \multirow{2}{*}{$\begin{array}{l}\text { Overall change } \\
(\%)^{*}\end{array}$} & \multicolumn{2}{|c|}{$\%$ Change associated with health reform $\dagger$} \\
\hline & Before reform & After reform & & Mean $(95 \% \mathrm{Cl})$ & P value $\ddagger$ \\
\hline \multicolumn{6}{|c|}{ Total knee and hip replacements } \\
\hline All & $29.0(28.5$ to 29.4$)$ & 35.5 (35.0 to 36.0$)$ & 22.4 & 4.7 (2.3 to 7.1$)$ & $<0.001$ \\
\hline \multicolumn{6}{|l|}{ Race/ethnicity: } \\
\hline White, non-Hispanic & 30.1 (29.6 to 30.6$)$ & 37.3 (36.7 to 37.8$)$ & 23.9 & $2.8(1.3$ to 4.4$)$ & 0.03 \\
\hline Black, non-Hispanic & 28.1 (26.0 to 30.2 ) & 37.9 (35.7 to 40.1$)$ & 34.9 & $11.4(7.2$ to 15.8$)$ & 0.03 \\
\hline Hispanic & 12.9 (11.4 to 14.5$)$ & 17.9 (16.4 to 19.4$)$ & 38.8 & 37.9 (31.8 to 44.2$)$ & $<0.001$ \\
\hline \multicolumn{6}{|c|}{ Zip code median income: } \\
\hline Low & 25.5 (24.6 to 26.4$)$ & 33.3 (32.3 to 34.2$)$ & 30.6 & 7.7 (1.9 to 13.8$)$ & $<0.001$ \\
\hline Medium & 28.9 (28.0 to 29.8 ) & 35.1 (34.1 to 36.0 ) & 21.5 & $3.7(-0.2$ to 7.7$)$ & 0.12 \\
\hline High & $30.4(29.8$ to 31.1$)$ & 36.5 (35.9 to 37.2$)$ & 20.1 & $4.7(3.0$ to 6.4$)$ & $<0.001$ \\
\hline \multicolumn{6}{|c|}{ Total knee replacements } \\
\hline All & $17.5(17.2$ to 17.9$)$ & 21.8 (21.4 to 22.2 ) & 24.6 & $5.3(2.5$ to 8.6$)$ & $<0.001$ \\
\hline \multicolumn{6}{|l|}{ Race/ethnicity: } \\
\hline White, non-Hispanic & $18.0(17.6$ to 18.4$)$ & 22.5 (22.1 to 22.9$)$ & 25.0 & $3.5(0.3$ to 6.7$)$ & 0.03 \\
\hline Black, non-Hispanic & 18.5 (16.8 to 20.2$)$ & 26.1 (24.3 to 28.0$)$ & 41.1 & 18.7 (12.4 to 25.5$)$ & 0.01 \\
\hline Hispanic & 9.9 (8.6 to 11.3$)$ & $13.6(12.3$ to 15.0$)$ & 37.4 & 33.0 (32.0 to 34.1$)$ & $<0.001$ \\
\hline \multicolumn{6}{|c|}{ Zip code median income: } \\
\hline Low & $16.7(16.0$ to 17.4$)$ & 21.5 (20.7 to 22.3 ) & 28.7 & $6.3(-0.2$ to 12.4$)$ & 0.06 \\
\hline Medium & 17.7 (17.0 to 18.4$)$ & 22.3 (21.6 to 23.1$)$ & 26.0 & 6.5 (3.7 to 9.4$)$ & 0.03 \\
\hline High & $17.7(17.2$ to 18.2$)$ & 21.6 (21.1 to 22.1$)$ & 22.0 & $5.4(2.7$ to 8.1$)$ & 0.01 \\
\hline \multicolumn{6}{|c|}{ Total hip replacements } \\
\hline All & 11.5 (11.2 to 11.8$)$ & $13.7(13.4$ to 14.0$)$ & 19.1 & $3.9(0.1$ to 7.8$)$ & 0.05 \\
\hline \multicolumn{6}{|l|}{ Race/ethnicity: } \\
\hline White, non-Hispanic & 12.1 (11.8 to 12.5$)$ & $14.8(14.4$ to 15.1$)$ & 22.3 & $2.1(-1.4$ to 5.8$)$ & 0.28 \\
\hline Black, non-Hispanic & 9.6 (8.4 to 10.8$)$ & 11.8 (10.6 to 13.0$)$ & 22.9 & $-1.8(-7.1$ to 3.8$)$ & 0.84 \\
\hline Hispanic & $3.0(2.3$ to 3.7$)$ & 4.2 (3.5 to 5.0$)$ & 40.0 & $49.8(26.7$ to 77.1$)$ & 0.01 \\
\hline \multicolumn{6}{|c|}{ Zip code median income: } \\
\hline Low & $8.8(8.3$ to 9.4$)$ & 11.7 (11.1 to 12.3$)$ & 33.0 & 10.6 (4.9 to 16.5$)$ & 0.02 \\
\hline Medium & $11.2(10.6$ to 11.8$)$ & 12.7 (12.2 to 13.3$)$ & 13.4 & $-0.3(-6.5$ to 6.4$)$ & 0.94 \\
\hline High & 12.7 (12.3 to 13.1$)$ & 14.9 (14.5 to 15.4$)$ & 17.3 & $4.2(-2.8$ to 11.7$)$ & 0.10 \\
\hline
\end{tabular}

${ }^{*}$ Overall change is $\%$ change between procedure rates before and after reform.

†Change associated with MA health reform obtained from difference-in-differences Poisson regression model to adjust for difference secular changes (based on comparison with New Jersey, New York, and Pennsylvania) with fixed effects for quarter and state. Standard errors corrected for clustering within states.

$\ddagger P$ value associated with null hypothesis of no change in procedure use associated with reform. 
Table 3| Proportion (percentage) of knee and hip replacement procedures carried out in safety net hospitals before and after health reform in Massachusetts

\begin{tabular}{|c|c|c|c|c|}
\hline & \multirow[b]{2}{*}{ Before reform $(95 \% \mathrm{Cl})$} & \multicolumn{2}{|c|}{ After reform } & \multirow{2}{*}{$\begin{array}{l}\text { Mean } \% \text { change in share in safety } \\
\text { net hospitals after reform }+(95 \% \\
\mathrm{Cl})\end{array}$} \\
\hline & & Observed & Mean expected ${ }^{\star}(95 \% \mathrm{CI})$ & \\
\hline All & 12.7 (12.2 to 13.3$)$ & 12.1 & 13.1 (12.9 to 13.3$)$ & $-1.0(-1.4$ to -0.6$)$ \\
\hline \multicolumn{5}{|l|}{ Race/ethnicity: } \\
\hline White, non-Hispanic & 11.5 (10.9 to 12.0$)$ & 10.7 & 11.7 (11.4 to 11.9$)$ & $-1.0(-1.4$ to -0.6$)$ \\
\hline Black, non-Hispanic & 30.4 (26.9 to 33.9 ) & 28.3 & 29.5 (28.6 to 30.4$)$ & $-1.2(-3.7$ to 1.3$)$ \\
\hline Hispanic & 30.3 (25.0 to 35.7 ) & 22.0 & 28.4 (26.8 to 30.0$)$ & $-6.4(-9.8$ to -3.0$)$ \\
\hline \multicolumn{5}{|c|}{ Zip code median income: } \\
\hline Low & 25.3 (23.7 to 26.9 ) & 22.8 & 26.7 (25.9 to 27.4 ) & $-3.9(-5.0$ to -2.8$)$ \\
\hline Medium & 12.9 (11.9 to 14.0$)$ & 11.6 & $12.8(12.5$ to 13.1$)$ & $-1.2(-2.0$ to -0.4$)$ \\
\hline High & $8.0(7.4$ to 8.6$)$ & 8.1 & 8.1 (7.9 to 8.2$)$ & $0.0(-0.5$ to 0.5$)$ \\
\hline
\end{tabular}

*Estimated from logistic regression model of receipt of procedure at safety net hospital with covariates of patient age, sex, Charlson comorbidity score, and difference in distance between residence zip code to nearest safety net hospital and to nearest non-safety-net hospital.

†Difference between observed and expected \% share of knee and hip replacement procedures after reform in safety net hospitals. 


\section{Figure}
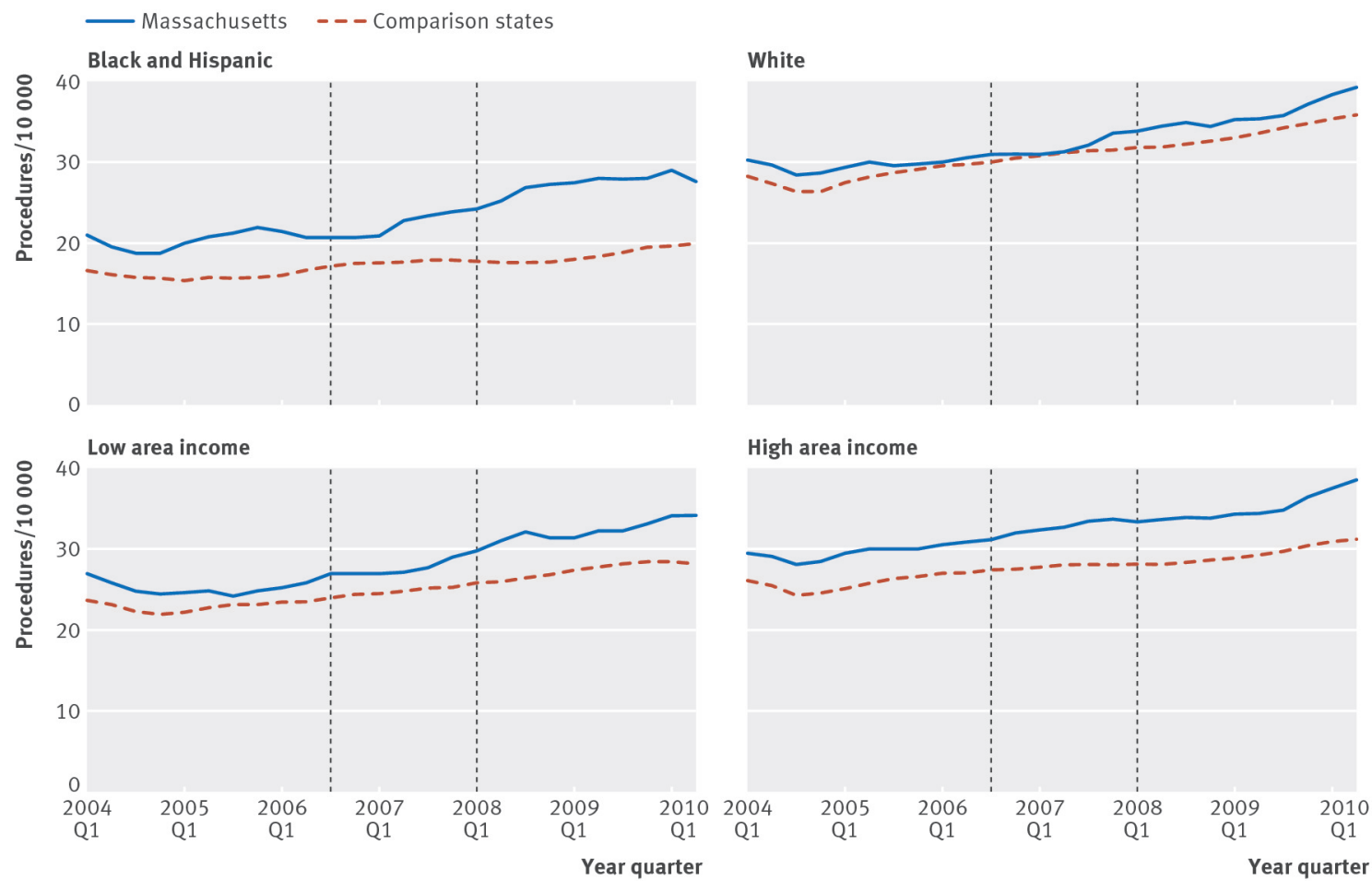

Procedure rates for knee and hip replacement in people aged 40-64 in Massachusetts and comparison states, 2004-10. Because of seasonal trends in procedure use, we obtained seasonally adjusted procedure rate for each quarter, defined as moving average of four quarters. We compared time trend in procedure rate before reform between Massachusetts and comparison states with log linear time series regression model. Results indicated that rates in Massachusetts after reform rose faster among black and Hispanic people $(\mathrm{P}<0.001)$ and residents of low income areas $(\mathrm{P}<0.01)$ but at similar rates among white people and residents of high income areas 